\title{
Preparation and Characterization of ZGDHu-1 Nanoparticles
}

\author{
Lei Shi", Yong-Li Liu, Kun-Kun Wu, and Rui-Yang Si \\ Chemical Pharmaceutical Research Institute, Taizhou Vocational \& Technical College, Taizhou, P. R. China.
}

e-mail: 36013107@qq.com

\begin{abstract}
The purpose of this study was to establish the preparation process of ZGDHu-1 nanoparticles and to study its physicochemical properties. The nanoparticles were prepared using a high-pressure homogenization method. Natural phospholipid and soybean oil were used as drug carriers, and Cremophor EL was used as surfactant. The resulting nanoparticles had a spherical shape, with a mean \pm SD particle size of $106.8 \pm 15.9 \mathrm{~nm}$, polydispersity index (PDI) of $0.136 \pm 0.011$, zeta potential of $-63.97 \pm 3.57 \mathrm{mV}$, encapsulation efficiency of $86.95 \%$, and in vitro cumulative release rate of $84.67 \%$ in 24 hours. The nanoparticle preparation process was simple and reproducible. The nanoparticles had good physicochemical properties and in vitro release was improved by increasing solubility. This study provides a method for improving in vitro release for development of ZGDHu-1 nanoparticle formulations.
\end{abstract}

KEYWORDS: ZGDHu-1, nanoparticles, entrapment efficiency, in vitro drug release, dissolution

\section{INTRODUCTION}

T etrazine derivatives have high potential as antiviral and antitumor agents (1). ZGDHu-1 is a symmetric tetrazine compound ( $\mathrm{N}, \mathrm{N}$-di-(m-methylphenyl)3,6-dimethyl-1,4-dihydro-1,2,4,5-tetrazine-1,4dicarboamide), synthesized by Wei-xiao Hu (2). Antitumor effects include anti-leukemia cells, proliferation and suppression of pancreatic cancer cells. Potential therapeutic effects include promotion of apoptosis of chronic lymphocytic leukemia (CLL) cells for treatment of lung cancer, a synergistic effect on apoptosis of CLL cells with fludarabine, and excellent results in treatment of acute myeloid leukemia (3-8).

At present, there are scarce reports in scientific literature about preparation of ZGDHu-1 formulations. Early studies show that ZGDHu-1 is insoluble in water and freely soluble in ethyl acetate and ethanol. These behaviors result in rather low dissolution and absorption rates, which have limited its clinical application.

Nanostructured materials display unusual chemical and physical properties that differ from bulk materials and are promising for fabrication of novel nano-devices (9). The preparation of nanoparticle formulations is one of the main topics in nanotechnology (10). Nanoparticles are composed of high polymer materials with a spherical framework. Nanoparticles can be prepared either by polymerization of emulsified monomers or by dispersion of preformed polymers (11). There are various methods developed to prepare drug-loaded nanoparticles depending on the physicochemical properties of the polymers and drugs (12). Particle size generally varies from 1 to $1000 \mathrm{~nm}$ (13). The nanoparticle drug delivery system can be improved by increasing dissolvability and bioavailability, reducing drug toxicity, changing the in vivo distribution, and controlling the drug release, among others (14-16).

The purpose of the present study was to establish a preparation method for ZGDHu-1 nanoparticle formulations and to increase the in vitro drug release rate. This study can provide a reference for research and development of new nanoparticle dosage forms.

\section{MATERIALS AND METHODS}

\section{Materials}

ZGDHu-1 was homemade and its purity was $99.64 \%$. Highpurity natural phospholipid was purchased from Shanghai Advanced Vehicle Technology Co., Ltd. (Shanghai, China). Soybean oil was obtained from Yihai Kerry (Shanghai, China). Cremophor EL and lactose anhydrous were analytically pure and obtained from Aladdin Biochemical Technology Co., Ltd. (Shanghai, China). Sephadex G-50

* Corresponding author. 
(100-200 mesh) was from Taizhou Sijiashenhua Plastic Co. (Taizhou, China). Methyl alcohol was chromatographically pure; mannitol and all other chemical reagents were analytically pure. Dialysis bags were from Union Carbide Corporation (Houston, TX, USA) with a molecular weight cut-off (MWCO) of 8-14 KD.

\section{Preparation of ZGDHu-1 Nanoparticles}

ZGDHu-1 nanoparticles were prepared by a high-pressure homogenizing method with one oil and one aqueous phase during the procedure. The natural phospholipid and soybean oil were mixed and dissolved in ethyl acetate as the oil phase. Cremophor EL surfactant was dissolved in water by ultrasound as the aqueous phase. ZGDHu-1 was dissolved in the oil phase. The oil phase was slowly added into the aqueous phase, and the mixture was stirred with a magnetic stirrer until emulsified. A clear nanoparticle solution was obtained after the emulsion was cycled six times and homogenized by a high-pressure homogenizer (AH-100D, ATS, Canada). The primary pressure was set at approximately 110 bars, and the secondary pressure was set at approximately 1250 bars. The blank nanoparticles were prepared by the same procedure.

The proportions of natural phospholipid, soybean oil, and Cremophor EL for orthogonal experiments with three factors and three levels are shown in Table 1. After single-factor experiments, the orthogonal experiments were designed, including polydispersity index (PDI) as an estimated index, the mass ratio of drug to natural phospholipids (A), mass ratio of drug to soybean oil (B), and mass concentration of surfactant (C) as the three factors with three levels. Particle size distribution is concentrated with the decrease of PDI. A smaller PDI means a better level of the corresponding factor. The influence of different factors was studied and the optimum formulation was obtained by processing and analyzing the PDI.

Table 1. Three Factors and Three Levels of Orthogonal Experimental Design

\begin{tabular}{|c|c|c|c|}
\hline Level & $\begin{array}{c}\text { A } \\
\text { (ZGDHu-1: } \\
\text { phospholipid) }\end{array}$ & $\begin{array}{c}\text { B } \\
\text { (ZGDHu-1: soybean } \\
\text { oil) }\end{array}$ & (Cremophor EL, \%) \\
\hline 1 & $1: 20$ & $1: 20$ & 0.7 \\
\hline 2 & $1: 25$ & $1: 25$ & 1.0 \\
\hline 3 & $1: 30$ & $1: 30$ & 1.3 \\
\hline
\end{tabular}

A: mass ratio of drug to natural phospholipids; B: mass ratio of drug to soybean oil; C: mass concentration of surfactant; Level represents the different values of three factors.

In addition, to obtain stable nanoparticle powder, mannitol and anhydrous lactose were dissolved in the nanoparticle solution as scaffolds, filled in a glass medicine bottle, then freeze dried by a vacuum freeze drier (LGJ10C, Sihuan, Beijing, China). The freeze-drying conditions consisted of primary drying at a shelf temperature of -45 ${ }^{\circ} \mathrm{C}$ for $10 \mathrm{~h}$ and secondary drying at $25^{\circ} \mathrm{C}$ for $3 \mathrm{~h}, 0{ }^{\circ} \mathrm{C}$ for $2 \mathrm{~h}, 10^{\circ} \mathrm{C}$ for $4 \mathrm{~h}$, and $30{ }^{\circ} \mathrm{C}$ for $8 \mathrm{~h}$. Chamber vacuum pressure was below $20 \mathrm{~Pa}$ throughout the drying cycle (17). The lyophilized powder was stored airtight at room temperature and dissolved in purified water, with shaking if needed.

\section{Morphology, Particle Size, PDI, and Zeta Potential Measurements}

The morphology of drug-loaded nanoparticles was determined by a scanning electron microscope (SEM) (S-4800, Hitachi, Japan) with a negative stain (phosphotungstate sodium [2\% W/V]). The mean particle size, size distribution, and PDI were measured by a laser light scattering meter (S90, Malvern Panalytical, Malvern, UK). The zeta potential was measured by a potentiometer (Delsa Nano C, Beckman Coulter, Brea, CA, USA). The lyophilized powder was redissolved and diluted to the appropriate concentration for determination.

\section{High-Performance Liquid Chromatography (HPLC) Analysis}

The HPLC system used for analysis was 1220 Infinity LC (Agilent, Germany) with Agilent ZORBAX SB-C18 columns $(4.6 \mathrm{~mm} \times 150 \mathrm{~mm}, 5 \mu \mathrm{m})$. The HPLC system was run at $35{ }^{\circ} \mathrm{C}$, the mobile phase was water:methanol $(8: 92 \mathrm{v} / \mathrm{v})$, the flow rate was $1.0 \mathrm{~mL} / \mathrm{min}$, the detection wavelength was $270 \mathrm{~nm}$, the injection volume was $20-\mu \mathrm{L}$, and the retention time of ZGDHu-1 was $2.8 \mathrm{~min}$.

\section{Entrapment Efficiency Analysis}

ZGDHu-1 and drug-loaded nanoparticles were separated using Sephadex G-50 column (Xingguang, Taizhou, China) chromatography. The drug-loaded nanoparticles were demulsified by ultrasound. Sephadex G-50 (3.3 g) was swelled for $24 \mathrm{~h}$ in purified water, boiled for $30 \mathrm{~min}$, cooled to room temperature, then filled into the column (column length $15 \mathrm{~cm}$, inner diameter $1 \mathrm{~cm}$ ). The column was flushed for $2 \mathrm{~h}$ with purified water at $1 \mathrm{~mL} / \mathrm{min}$. The injection volume of ZGDHu-1 nanoparticles solution was $1.0 \mathrm{~mL}$. Elution with purified water was set at $1 \mathrm{~mL} / \mathrm{min}$, collecting the eluent in sample tubes as $1 \mathrm{~mL}$ per tube for the first 20 tubes, then eluted with $70 \%$ ethanol solution. The collect eluent was transferred from the sample tube to a $5-\mathrm{mL}$ volumetric flask, adding mobile phase to dissolve, then the final solution was ultrasonicated and measured by HPLC.

The elution curve is shown in Figure 1. The drug-loaded nanoparticles were eluted in tubes $1-20$, and free 
ZGDHu-1 was eluted in tubes 21-50. The amount of drug entrapment in nanoparticles was detected, and the entrapment efficiency was calculated as shown in Equation 1:
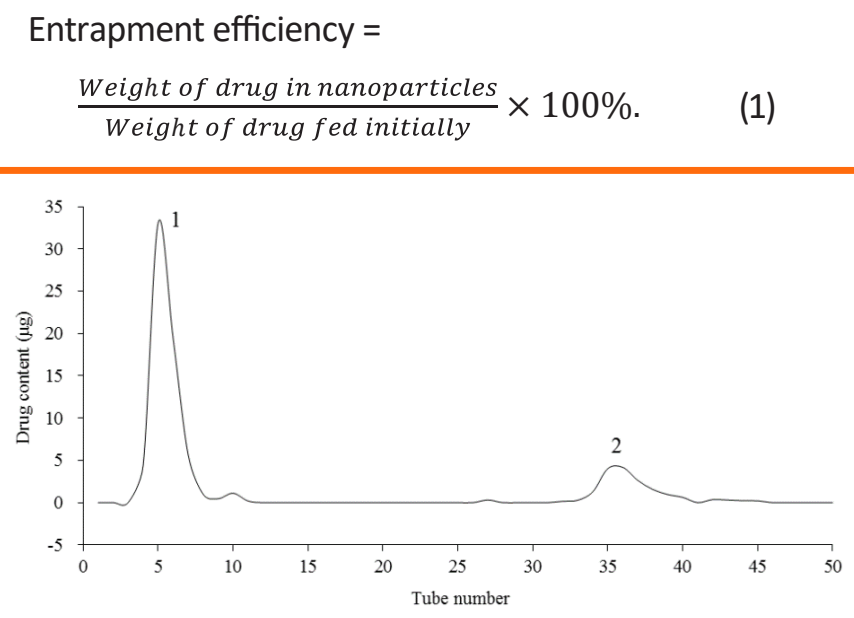

Figure 1. Elution curve of ZGDHu-1 nanoparticles. 1: drug-loaded nanoparticle; 2: ZGDHu-1.

\section{Differential Scanning Calorimeter (DSC)}

The thermograms were measured on differential scanning calorimeter (DSC) (DSC-100, Dazhan, Nanjing, China) at a heating rate of $10{ }^{\circ} \mathrm{C}$ per min with the temperature range from 50 to $300{ }^{\circ} \mathrm{C}$. The powder was filled into an aluminum crucible and scanned with the DSC.

\section{In Vitro Drug Release Studies}

The dialysis bag was boiled for $5 \mathrm{~min}$, flushed for $2 \mathrm{~min}$ with $60-^{\circ} \mathrm{C}$ distilled water, and cooled. Drug-loaded nanoparticle solution (7 mL, $1.0 \mathrm{mg}$ ZGDHu-1) was added to the dialysis bags for dissolution experiments. The bags were inserted into dissolution vessels with $300 \mathrm{~mL}$ phosphate buffer solution ( $\mathrm{pH} 6.8)$. The stirring rate was $50 \mathrm{rpm}$, the solution temperature was $37^{\circ} \mathrm{C}$. Samples $(5$ $\mathrm{mL}$ ) were extracted from the bulk solution surrounding the dialysis bag at $0.5,4,8,12,20$, and $24 \mathrm{~h}$, and an equal volume of phosphate buffer solution was added at each interval.

The resulting sample was filtered with a 0.45$\mu \mathrm{m}$ membrane (Xinya, Shanghai, China). ZGDHu-1 concentrations in the sample were quantified by HPLC. In vitro cumulative release rate was calculated with Equation 2:

Cumulative release $=$

$$
\frac{\text { Drug concentration } \times \text { Volume of bulk solution }}{\text { Weight of drug fed initially }} \times 100 \% \text {. }
$$

In addition, ZGDHu-1 isolated crystals were added in aqueous solution of Cremophor EL with a mass percent concentration of $1.3 \%$. The suspension was studied by the same dialysis method.

\section{RESULTS AND DISCUSSION}

\section{Preparation Method of Nanoparticles}

Results of PDI and variance analyses from orthogonal experiments are shown in Table 2 . The results showed that the influence degree of three factors was $A>C>B$, and the optimum formulation was $A 2, B 1$, and $C 3$. The optimized formula was as follows: mass ratio of drug to natural phospholipids (A) was 1:25, mass ratio of drug to soybean oil (B) was 1:20, and mass concentration of surfactant $(C)$ was $1.3 \%$. According to this formula, three batches were prepared. The PDI ranged from 0.120 to 1.149, and the particle size ranged from 105.4 to 200.9 $\mathrm{nm}$, resulting in high reproducibility of the preparation process. The nanoparticles were prepared with a small PDI and particle size. The results of these experiments show that the process was simple and reproducible.

Table 2. PDI and Variance Analyses of Orthogonal Experiments

\begin{tabular}{|c|c|c|c|c|c|}
\hline $\begin{array}{c}\text { Experiment } \\
\text { No. }\end{array}$ & A & B & C & & PDI \\
\hline 1 & A1 & B1 & C1 & & 0.279 \\
\hline 2 & A1 & B2 & $\mathrm{C} 2$ & & 0.160 \\
\hline 3 & A1 & B3 & C3 & & 0.129 \\
\hline 4 & $\mathrm{~A} 2$ & B1 & $\mathrm{C} 2$ & & 0.199 \\
\hline 5 & A2 & B2 & C3 & & 0.211 \\
\hline 6 & $\mathrm{~A} 2$ & B3 & $\mathrm{C} 1$ & & 0.151 \\
\hline 7 & A3 & B1 & C3 & & 0.267 \\
\hline 8 & A3 & B2 & $\mathrm{C} 1$ & & 0.606 \\
\hline 9 & A3 & B3 & $\mathrm{C} 2$ & & 0.790 \\
\hline Ij & 0.189 & 0.248 & 0.345 & & \\
\hline IIj & 0.187 & 0.326 & 0.383 & & \\
\hline IIIj & 0.554 & 0.357 & 0.202 & & \\
\hline $\mathrm{Rj}$ & 0.367 & 0.109 & 0.181 & & \\
\hline Source & sS & $d f$ & $\begin{array}{l}\text { Mean } \\
\text { SS }\end{array}$ & F-value & $p$-value \\
\hline A & 0.268 & 2 & 0.134 & 14.358 & 0.002 \\
\hline B & 0.019 & 2 & 0.009 & 1.000 & 0.410 \\
\hline C & 0.054 & 2 & 0.027 & 2.918 & 0.112 \\
\hline Error & 0.082 & 8 & & & \\
\hline
\end{tabular}

A: mass ratio of drug to natural phospholipids; $B$ : mass ratio of drug to soybean oil; C: mass concentration of surfactant; PDI: polydispersity index; Ij: average PDI of level 1; Ilj: average PDI of level 2; IIIj: average PDI of level 3; Rj: range of average PDI from level 1-3; SS: sum of squares of difference of the mean; $d f$ : degrees of freedom of orthogonal test. 
Thus, the optimal ZGDHu-1 nanoparticle preparation method was as follows. Two phases of oil and aqueous were included in the preparation procedure $-100 \mathrm{mg}$ of natural phospholipid and $80 \mathrm{mg}$ of soybean oil were mixed and dissolved in $15 \mathrm{~mL}$ of ethyl acetate as the oil phase, and $364 \mathrm{mg}$ of Cremophor EL as surfactant was dissolved by ultrasound in $28 \mathrm{~g}$ of purified water as the aqueous phase. Then $4 \mathrm{mg}$ of ZGDHu-1 was dissolved in the oil phase. The phases were mixed slowly and stirred with a magnetic stirrer until emulsified. The clear nanoparticle solution was obtained after the emulsion was cycled and homogenized by a high-pressure homogenizer. The blank nanoparticles were prepared as described before.

There are many ways to prepare nanoparticles reported in the literature, such as emulsion solvent evaporation, emulsion-diffusion, and solvent displacement precipitation, among others (18). However, high-pressure homogenizers are widely used in the pharmaceutical, chemical, and food industries (19). In the homogenization process, the emulsion was recycled, sheared, and impacted until nanoparticles were formed. In addition, ethyl acetate as a less toxic and good solubility solvent was volatilized.

Previous studies have shown that natural phospholipids and soybean oil could well encapsulate ZGDHu-1 (17). Cremophor EL (chosen as surfactant) is widely used in anticancer drugs for increasing bioavailability without Tween's hemolysis. Thus, the emulsion solvent evaporation method was chosen to prepare ZGDHu-1 nanoparticles by a high-pressure homogenization method.

\section{Morphology, Particle Size, PDI, and Zeta Potential}

The SEM photographs and particle size distribution of prepared nanoparticles are shown in Figure 2. SEM photographs show that most drug-loaded nanoparticles had a discrete spherical shape. The mean particle size was $106.8 \pm 15.9 \mathrm{~nm}$, and mean PDI was $0.136 \pm$ 0.011 . The mean zeta potential was $-63.97 \pm 3.57 \mathrm{mV}$, indicating surface charge greater than $-25 \mathrm{mV}$. Thus, the nanoparticles are not easy to conglomerate.

\section{Entrapment Efficiency}

Entrapment efficiency was $86.95 \%$ in the nanoparticle solution and $81.60 \%$ in the freeze-dried nanoparticle powder. In both cases, the entrapment quantity of nanoparticles was significant.

During the elution process, ZGDHu-1 nanoparticles are easy to separate for bigger particle size and better water solubility; the free ZGDHu-1 is eluted later due to poor water solubility, easily soluble in ethanol, and has a small molecular weight that can enter the gel. Based on these characteristics, purified water was used as the mobile solution for nanoparticles, followed by ethanol solution for the free drug to ensure the isolation effect.

\section{DSC}

Figure 3 shows the DSC thermograms for ZGDHu-1 substance, blank nanoparticles lyophilized powder, and drug-loaded nanoparticles lyophilized powder. The thermogram of ZGDHu-1 substance showed an endothermic peak at $210-270{ }^{\circ} \mathrm{C}$, corresponding to its melting temperature; this endothermic peak was not detected in the thermograms for drug-loaded nanoparticles lyophilized powder. Thus, ZGDHu-1 was

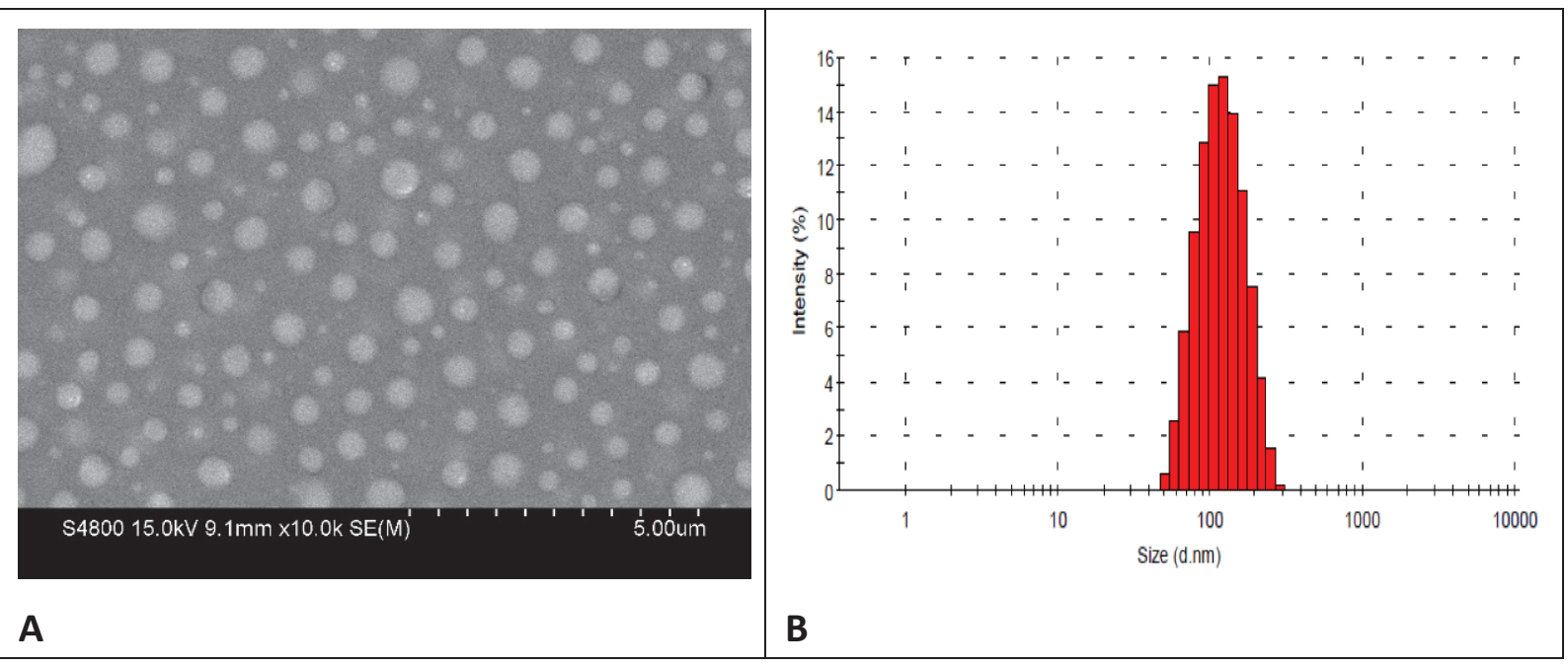

Figure 2. SEM photograph (A) and particle size distribution (B) of drug-loaded nanoparticles. SEM: scanning electron microscope. 
dispersed in a molecular state or amorphous state in drug-loaded nanoparticles.

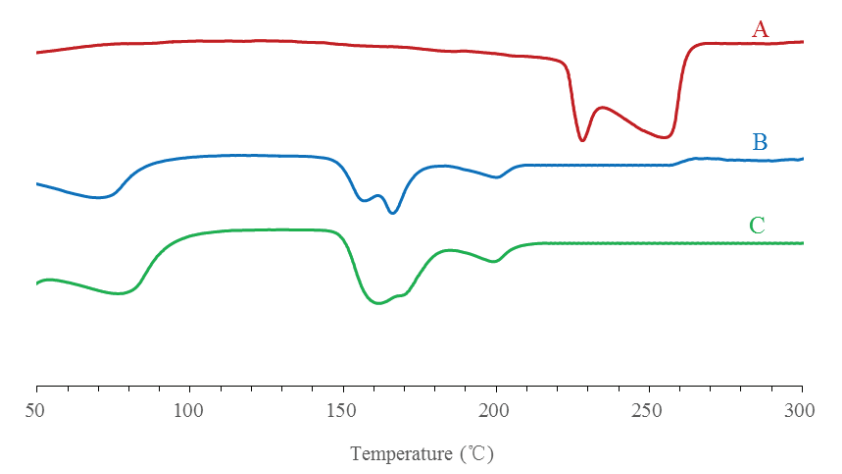

Figure 3. Differential scanning calorimeter (DSC) of the samples: ZGDHu-1 (A); blank lyophilized nanoparticles (B), and drug-loaded nanoparticles lyophilized powder (C).

\section{In Vitro Release}

Figure 4 shows the in vitro cumulative drug release rate for ZGDHu-1 in phosphate buffer solution (pH 6.8) $(84.67 \%$ in 24 hours). The drug-loaded nanoparticles released faster than the drug isolate crystals.

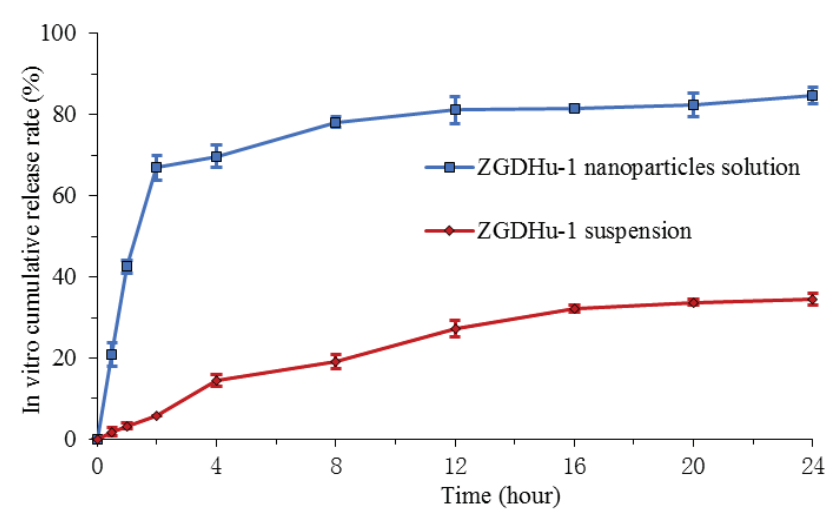

Figure 4. Drug in vitro release profiles of ZGDHu-1 suspension with Cremophor EL and ZGDHu-1 nanoparticles solution. Data are presented as mean $\pm S D(n=3)$.

In vitro drug release behavior was studied using a dialysis bag. The dialysis method is used widely to determine the in vitro release behavior of nanoparticle drug delivery systems. The drug released from nano-carriers first enters the solution inside the dialysis bag and then permeates through the dialysis membrane to reach the bulk solution outside the bag (20). The release rate is influenced by the concentration gradient between the inner bag and the outside bulk solution. The volume of the outside bulk solution was enough to dissolve the drug and was superior to that inside the bag. The diffusion rate was increased by using a larger pore-sized dialysis membrane and stirring.
The dissolution of a drug can be affected by many factors, such as water solubility and nanostructure of nanoparticles, among others. For the drug-loaded nanoparticles, the anti-cancer was encapsulated in the drug carrier. The water solubility of nanoparticles was superior to the insoluble drug substance, and the release rate was improved by increasing drug dispersion. From these results, two factors can be considered: the burst effect (drug adsorbed or embedded on the surface of nanoparticles is rapidly released), and the diffusion controlled-release effect (the encapsulated drug was slowly released from the drug carrier).

\section{CONCLUSION}

A series of drug-loaded nanoparticles of ZGDHu-1 was successfully prepared using a high-pressure homogenization method. The particle size and PDI were small, and the nanoparticles had a discrete spherical shape and good entrapment efficiency. These results show that natural phospholipid, soybean oil, and Cremophor EL are promising materials for preparing nanoparticles as drug carrier and surfactant. ZGDHu-1 nanoparticles have high water solubility. The in vitro drug release rate of nanoparticle formulation was better than that of the free drug substance. The preparation method was simple and reproducible. Future studies should focus on further development of ZGDHu-1 formulations.

\section{ACKNOWLEDGMENTS}

This project was financially supported by the Taizhou Science and Technology Project and the Zhejiang Public Welfare Technology Research Program of China.

\section{CONFLICT OF INTEREST}

The authors disclosed no conflicts of interest related to this article.

\section{REFERENCES}

1. Rao, G. W.; Guo, Y. M.; Hu, W. X. Synthesis, structure analysis, and antitumor evaluation of 3,6-dimethyl-1,2,4,5tetrazine-1,4-dicarboxamide derivatives. ChemMedChem. 2012, 7, 973-976. DOI: 10.1002/cmdc.201200109.

2. Hu, W. X. Synthesis of new type antitumor drug 3,6-dimethyl1,4-dihydro-1,2,4,5-tetrazine-1,4-dicarboamide. China Patent CN1223259A, July 21, 1999. http://pss-system.cnipa.gov.cn/ sipopublicsearch/inportal/i18n.shtml?params=902F004CA6108 4A284089435EAAEA94F59CC921A916ADCEB (accessed July 12, 2021).

3. Liu, J.; Qiu, L.; Xia, J.; Chen, S.; Yu, X.; Zhou, Y. ZGDHu-1 for cancer therapy. Oncol. Lett. 2017, 14, 6334-6340. DOI: 10.3892/ ol.2017.7096.

4. Qiu, L. N.; Zhou, Y. L.; Wang, Z. N.; Huang, Q.; Hu, W. X. ZGDHu-1 
promotes apoptosis of chronic lymphocytic leukemia cells. Int. J. Oncol. 2012, 41, 533-540. DOI: 10.3892/ijo.2012.1467.

5. Chen, S. F.; Xia, J.; Lv, Y. P.; Liu, J. L.; Li, W. X.; Yu, X. P.; Hu, W. X.; Zhou, Y. L. N. N,N'-di-(m-methylphenyi)-3,6-dimethyl-1,4-dihydro1,2,4,5-tetrazine-1,4-dicarboamide (ZGDHu-1) suppresses the proliferation of PANC-1 pancreatic cancer cells via apoptosis and G2/M cell cycle arrest. Oncol. Rep. 2015, 33, 1915-1921. DOI: 10.3892/or.2015.3803.

6. Shen, X. F.; Wu, Z. Chen, S.F.; Chen, Y.; Xia, J.; Lv, Y.P.; Zhou, Y.L. Introduction of G2/M phase arrest and apoptosis by ZGDHU-1 in A549 an Rerf-LC-MA lung cancer cells. Oncol. Lett. 2016, 12, 989-994. DOI: 10.3892/ol.2016.4697.

7. Qiu, L.; Liu, J.; Wang, Z.; Hu, W.; Huang, Q.; Zhou, Y. ZGDHu-1 and fludarabine have a synergistic effect on apoptosis of chronic lymphocytic leukemia cells. Oncol. Rep. 2015, 34, 1239-1248. DOI: 10.3892/or.2015.4115.

8. Xia, J.; Chen, S. F.; Lv, Y. P.; Lu, L. N.; Hu, W. X.; Zhou, Y. L. ZGDHu-1 induces $\mathrm{G}_{2} / \mathrm{M}$ phase arrest and apoptosis in Kasumi-1 cells. Mol. Med. Rep. 2015, 11, 3398-3404. DOI: 10.3892/mmr.2015.3160.

9. Seo, W.S.; Jo, H.H.; Lee, K.; Park, J.T. Preparation and optical properties of highly crystalline, colloidal, and size-controlled indium oxide nanoparticals. Adv. Mater. 2003, 15, 795-797. DOI: 10.1002/adma.200304568.

10. Nalwa, H. Handbook of Nanostructured Materials and Nanotechnology, Vol 1; Academic Press. 2000; pp 631-639. DOI: 10.1016/B978-012513760-7/50066-6.

11. Isaacs, P. K.; Edelhauser, H. A. Influence of polymer solubilization on latex behavior. J. Appl. Polym. Sci. 1966, 10, 171-183. DOI: 10.1002/app.1966.070100113.

12. Ge, H. X.; Hu, Y.; Yang, S. C.; Jiang, X. Q.; Yang, C. Z. Preparation, characterization, and drug release behaviors of drug-loaded E-caprolactone/L-lactide copolymer nanoparticles. J. Appl. Polym. Sci. 2000, 75, 874-882. DOI: 10.1002/(SICI)10974628(20000214)75:7<874::AID-APP3>3.0.CO;2-G.
13. Yadav, V.; Mahor, A.; Prajapati, S.; Alok, S.; Amita, V.; Kumar, A.; Gupta, N.; Kumar, S. Solid lipid nanoparticles (SLN): Formulation by high pressure homogenization. World J. Pharm. Pharm. Sci. 2014, 3, 1200-1213.

14. Patra, J. K.; Das, G.; Fraceto, L. F.; Campos, E. V. R.; RodriguezTorres, M. D. P.; Acosta-Torres, L. S.; Diaz-Torres, L. A.; Grillo, R.; Swamy, M. K.; Sharma, S.; et al. Nano based drug delivery systems: recent developments and future prospects. J. Nanobiotechnology. 2018, 16, 71. DOI: 10.1186/s12951-018-0392-8.

15. Mirza, A. Z.; Siddiqui, F. A. Nanomedicine and drug delivery: a mini review. Int. Nano Lett. 2014, 4, 94. DOI: 10.1007/s40089-0140094-7.

16. Abdelwahab, S. I.; Sheikh, B. Y.; Taha, M. M.; How, C. W.; Abdullah, R.; Yagoub, U.; El-Sunousi, R.; Eid, E. E. Thymoquinone-loaded nanostructured lipid carriers: preparation, gastroprotection, in vitro toxicity, and pharmacokinetic properties after extravascular administration. Int. J. Nanomedicine. 2013, 8, 2163-2172. DOI: 10.2147/IJN.S44108.

17. Shi, L. A compound of ZGDHu-1 nanoparticles and its preparation method.. China Patent CN105902504A, August 31, 2016. http:// pss-system.cnipa.gov.cn/sipopublicsearch/inportal/i18n.shtml?p arams $=902$ F004CA61084A284089435EAAEA94F59CC921A916A DCEB (accessed July 12, 2021).

18. Pal, S. L.; Jana, U.; Manna, P. K.; Manna; Mohanta, G. P.; Manavalan, R. Nanoparticle: An overview of preparation and characterization. J. Appl. Pharm. Sci. 2011, 1, 228-234.

19. Köhler, K.; Aguilar, F. A.; Hensel, A.; Schubert, K.; Schubert, H.; Schuchmann, H. P. Design of a microstructured system for homogenization of dairy products with high fat Content. Chem. Eng. Technol. 2007, 30, 1590-1595. DOI: 10.1002/ceat.200700266.

20. Solomon, D.; Gupta, N.; Mulla, N. S.; Shukla, S.; Guerrero, Y. A.; Gupta, V. Role of in vitro release methods in liposomal formulation development: challenges and regulatory perspective. AAPS J. 2017, 19, 6: 1669-1681. DOI: 10.1208/s12248-017-0142-0. 